\title{
Review of: "Regulation of major bacterial survival strategies by transcript sequestration in a membraneless organelle"
}

\author{
Semyon Nesterov
}

Potential competing interests: The author(s) declared that no potential competing interests exist.

The article of Szoke et al. (1) is an extension of their previous work about novel bacterial signaling cascade associated with the TmaR protein. The importance of this signaling cascade is hard to overestimate because it connects tyrosine phosphorylation with spatial regulation and sugar metabolism, thus controlling key processes essential for bacterial survival (2). Previously, it was found that TmaR accumulates in cell poles after phosphorylation. Cell poles are the main sites providing specific localization of cellular components in bacteria, which unlike eukaryotes have no classical membrane organelles. However, the exact physicochemical mechanisms of selective localization of both TmaR and other proteins associated with cell poles remained unknown. Taking into account the sharp increase over the past couple of years in the number of data on recently discovered principle of self-organization of biological systems liquid-liquid phase separation (LLPS), which is also found in bacteria (3-7), Szoke et al. reasonably suggested that LLPS may participate in TmaR functioning. This assumption was entirely logical, since TmaR caused the selective accumulation of transcripts in a limited area of the cell, which is very similar to the function of membraneless organelles formed by LLPS. It is well-known that LLPS is stimulated by proteins containing intrinsically disordered regions and computer prediction confirms that TmaR have rather long disordered domains. Subsequent in vitro studies of GFP-TmaR by Szoke et al. left no doubt that TmaR have the ability to undergo LLPS. Experiments on bacteria, including those using the FRAP method, also confirmed the formation of condensates. This raises the question that other processes associated with cell poles, such as the operation of the division system (8) are likely closely related to LLPS. Thus, the result obtained by Szoke et al. sheds light on the mechanisms of self-organization in bacteria, which is extremely important for the development of biotechnology and medicine.

Szoke et al. also showed that increased TmaR expression causes filamentation. This is a common phenomenon in condensates in the presence of proteins prone to oligomerization (9). A very interesting result is that this filaments penetrated from cell to cell, forming a visually uniform structure (Fig. 3 in (1)), despite the presence of membranes between them. This phenomenon confirms predictions that condensates (and apparently also aggregates formed from them) can spread across the membranes. This mechanism of transmembrane signaling by LLPS is proposed by Nesterov et al. (9). According to this model, the contact of the condensate with the membrane causes the formation of a bilayer raft, which in turn catalyzes phase separation on the other side of the membrane (and then maybe filamentation of 
some proteins in the condensate). The bridges between bacteria found by Szoke et al. could be formed in a similar way. In the case described by Szoke et al., filamentation process had clearly pathological nature and was observed only after overexpression of TmaR. This result raises a logical question: can the penetration of aggregates from cell to cell also occur in humans in a similar way, for example, during the aggregation of beta-amyloid? A quick literature search shows that such an effect is principally possible (10). If further research confirms this similarity of processes, it will make bacteria an interesting simple model for the study of some socially significant human diseases.

It should be noted that such a "transfer of the condensate" across the membrane can occur not only in pathological case, but also can play a significant signaling role under normal conditions of cell functioning. As the experiments showed, TmaR knockout prevents the formation of a biofilm, which is undoubtedly associated with impaired transcript accumulation in cell polars. However, it is tempting to suggest that the role of TmaR-condensates in biofilm formation is not limited to regulation of protein expression and distribution. Possibly, condensate spreading between bacteria upon their contacts is also an important factor for biofilm stabilization. The study of more detailed mechanisms of condensates participation in biofilm formation and signal exchange between bacteria requires further clarification.

Thus, the work of Szoke et al. certainly deserves attention and, as good research should, opens up new research perspectives.

\section{Bibliography}

1. Szoke, T., Albocher, N., Goldberger, O., Barsheshet, M., Nussbaum-Shochat, A., Wiener, R., Schuldiner, M. and Amster-Choder, O. (2022) Regulation of major bacterial survival strategies by transcript sequestration in a membraneless organelle. https://doi.org/10.1101/2022.01.06.475198.

2. Szoke, T., Albocher, N., Govindarajan, S., Nussbaum-Shochat, A. and Amster-Choder, O. (2021) Tyrosine phosphorylation-dependent localization of TmaR that controls activity of a major bacterial sugar regulator by polar sequestration. Proc. Natl. Acad. Sci. 118. https://doi.org/10.1073/pnas.2016017118.

3. Azaldegui, C. A., Vecchiarelli, A. G. and Biteen, J. S. (2020) The Emergence of Phase Separation as an Organizing Principle in Bacteria. Biophys. J. 0. https://doi.org/10.1016/j.bpj.2020.09.023.

4. Bollen, C., Dewachter, L. and Michiels, J. (2021) Protein Aggregation as a Bacterial Strategy to Survive Antibiotic Treatment. Front. Mol. Biosci. 8, 669664. https://doi.org/10.3389/fmolb.2021.669664.

5. Jin, X., Lee, J.-E., Schaefer, C., Luo, X., Wollman, A. J. M., Payne-Dwyer, A. L., Tian, T., Zhang, X., Chen, X., Li, Y., McLeish, T. C. B., Leake, M. C. and Bai, F. (2021) Membraneless organelles formed by liquid-liquid phase separation increase bacterial fitness. Sci. Adv. https://doi.org/10.1126/sciadv.abh2929.

6. Ladouceur, A.-M., Parmar, B. S., Biedzinski, S., Wall, J., Tope, S. G., Cohn, D., Kim, A., Soubry, N., ReyesLamothe, R. and Weber, S. C. (2020) Clusters of bacterial RNA polymerase are biomolecular condensates that assemble through liquid-liquid phase separation. Proc. Natl. Acad. Sci. 117, 18540-18549. https://doi.org/10.1073/pnas.2005019117.

7. Guilhas, B., Walter, J.-C., Rech, J., David, G., Walliser, N. O., Palmeri, J., Mathieu-Demaziere, C., 
Parmeggiani, A., Bouet, J.-Y., Le Gall, A. and Nollmann, M. (2020) ATP-Driven Separation of Liquid Phase Condensates in Bacteria. Mol. Cel/79, 293-303.e4. https://doi.org/10.1016/j.molcel.2020.06.034.

8. Raskin, D. M. and de Boer, P. A. J. (1999) Rapid pole-to-pole oscillation of a protein required for directing division to the middle of Escherichia coli. Proc. Natl. Acad. Sci. U. S. A. 96, 4971-4976.

9. Nesterov, S. V., Ilyinsky, N. S. and Uversky, V. N. (2021) Liquid-liquid phase separation as a common organizing principle of intracellular space and biomembranes providing dynamic adaptive responses. Biochim. Biophys. Acta BBA - Mol. Cell Res. 1868, 119102. https://doi.org/10.1016/j.bbamcr.2021.119102. 10. Domert, J., Rao, S. B., Agholme, L., Brorsson, A.-C., Marcusson, J., Hallbeck, M. and Nath, S. (2014) Spreading of amyloid- $\beta$ peptides via neuritic cell-to-cell transfer is dependent on insufficient cellular clearance. Neurobiol. Dis. 65, 82-92. https://doi.org/10.1016/j.nbd.2013.12.019. 